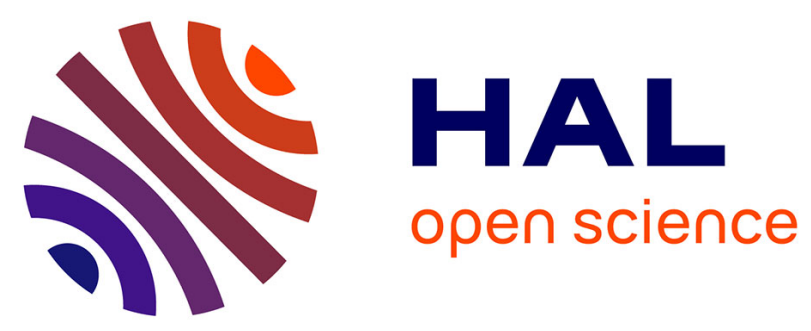

\title{
Identification of novel species of marine magnetotactic bacteria affiliated with Nitrospirae phylum
}

Xin-xin Qian, J Liu, Nicolas Menguy, Jinhua Li, François Alberto, Zhaojie Teng, Tian Xiao, Wenyan Zhang, Long-fei Wu

\section{- To cite this version:}

Xin-xin Qian, J Liu, Nicolas Menguy, Jinhua Li, François Alberto, et al.. Identification of novel species of marine magnetotactic bacteria affiliated with Nitrospirae phylum. Environmental Microbiology Reports, 2019, 11 (3), pp.330-337. 10.1111/1758-2229.12755 . hal-02333904

\section{HAL Id: hal-02333904 https://hal-amu.archives-ouvertes.fr/hal-02333904}

Submitted on 10 Feb 2020

HAL is a multi-disciplinary open access archive for the deposit and dissemination of scientific research documents, whether they are published or not. The documents may come from teaching and research institutions in France or abroad, or from public or private research centers.
L'archive ouverte pluridisciplinaire HAL, est destinée au dépôt et à la diffusion de documents scientifiques de niveau recherche, publiés ou non, émanant des établissements d'enseignement et de recherche français ou étrangers, des laboratoires publics ou privés. 


\section{Nitrospirae phylum}

3 Xin-Xin Qian ${ }^{\text {a,b,1 }}$, Jia Liu ${ }^{\text {b,d,1,2 }}$, Nicolas Menguy ${ }^{\text {b,c }}$, Jinhua Li ${ }^{\text {bee }}$, François Alberto ${ }^{\text {a,b }}$, Zhaojie Teng $^{d}$, Tian Xiao ${ }^{\text {b,d }}$, Wenyan Zhang ${ }^{\text {b,d,3 }}$, Long-Fei Wu ${ }^{\text {a,b,3 }}$

a Aix Marseille University, CNRS, LCB, Marseille, 13402, France

b International Associated Laboratory of Evolution and Development of Magnetotactic Multicellular Organisms (LIA-MagMC), CNRS-CAS, Marseille, 13402, France c Institut de Minéralogie, de Physique des Matériaux et de Cosmochimie, UMR 7590 CNRSSorbonne Université, F-75005 Paris. France

d CAS Key Laboratory of Marine Ecology and Environmental Sciences, Institute of

11 Oceanology, Chinese Academy of Sciences, Qingdao, 266071, China

e Key Laboratory of Earth and Planetary Physics, Institute of Geology and Geophysics, Chinese Academy of Sciences, Beijing, 100029, China

1 Contributed equally to this work

2 Current address: Key Laboratory of Earth and Planetary Physics, Institute of Geology and Geophysics, Chinese Academy of Sciences, Beijing, 100029, China

3 To whom correspondence should be addressed: Long-Fei WU, LCB, CNRS, 31 chemin Joseph Aiguier, 13402 Marseille cedex 20, France, 33-4-9116 4157, wu@imm.cnrs.fr; Wenyan ZHANG, CAS Key Laboratory of Marine Ecology and Environmental Sciences, 8289 8586, zhangwy@qdio.ac.cn 


\section{Summary}

Magnetotactic bacteria (MTB) are a group of Gram-negative bacteria characterized by synthesizing magnetosomes and swimming along geomagnetic field lines. Phylogenetically, they belong to different taxonomic lineages including Proteobacteria, Nitrospirae, Omnitrophica, Latescibacteria, and Planctomycetes phyla on the phylogenetic tree. To date, six Nitrospirae MTB phylotypes have been identified from freshwater or low salinity environments and described in the literature. Here we report the identification of two Nitrospirae MTB phylotypes collected, for the first time, from the marine environment. Both have a spherical morphology with a cell size of $\sim 5 \mu \mathrm{M}$ and similar motility but are different colors (black-brown and ivory-white) under the optic microscope. They synthesized bullet-shaped iron-oxide magnetosomes that were arranged in multiple bundles of chains. Moreover, the cytoplasm of the black-brown Nitrospirae MTB contained sulfur inclusions that conferred on cells a rough, granular appearance. Phylogenetic analysis based on their 16S rRNA gene sequences revealed that they are two novel species and cluster with the previously reported MTB affiliated with the phylum Nitrospirae, thus extending the distribution of Nitrospirae MTB from freshwater to the marine environment.

\section{Originality-Significance Statement}

Magnetotactic bacteria (MTB) consist of a phylogenetic, physiological and morphologic heterogeneous group of gram-negative microbes. They are distributed worldwide at the oxic-anoxic interface in chemically stratified water columns or sediments. To date, despite Nitrospirae being one of the major phyla of MTB, they have only been found in freshwater or 
low-salinity environments. Here we report the identification of two novel species of Nitrospirae MTB, from the marine habitat for the first time. They have a spherical morphology and are relatively big in size approximately $5 \times 3 \mu \mathrm{m}$. One contains abundant intracellular sulfur inclusions. This study shows the great diversity of Nitrospirae phylum and extends the distribution of Nitrospirae MTB into marine habitats.

\section{Introduction}

Magnetotactic bacteria (MTB) are a group of Gram-negative bacteria and exhibit the unique capacity of aligning and swimming along the geomagnetic field lines, a behavior referred to as magnetotaxis (Uebe and Schüler, 2016). This capacity is based on the intracellular biomineralization of magnetite or greigite magnetic nanocrystal chains that give MTB cells a net magnetic moment dipole. MTB present various morphotypes including cocci, spirilla, rod-shaped, vibrio, and more complex multicellular aggregates, and have been found in aquatic environments from freshwater to marine ecosystems (Blakemore, 1975; Bazylinski and Frankel, 2004; Lefèvre and Bazylinski, 2013). Phylogenetically, magnetotactic bacteria are members of several classes of the Proteobacteria phylum including the Alpha-, Gamma-, Delta-, Zeta-, candidate Lambda-, candidate Eta-classes, the Nitrospirae phylum, the candidate Omnitrophica phylum, the candidate Latescibacteria phylum and the Planctomycetes phylum (Lin et al., 2017a; Lin et al., 2018).

Compared with the Proteobacteria phylum, few MTB have been found to be phylogenetically affiliated with the phylum Nitrospirae and none of them have been cultured. This is consistent with the general difficulty in cultivating MTB due to their factitious 
physiological requirements (Bazylinski and Frankel, 2004). The first to be discovered and the most studied was Candidatus Magnetobacterium bavaricum (Spring et al., 1993). Ca. Magnetobacterium bavaricum is a large, rod-shaped bacterium ranging from 8 to $10 \mu \mathrm{m}$ in length, and contains 600-1000 bullet-shaped magnetite magnetosomes in 3-5 bundles of multiple chains (Jogler et al., 2010; Jogler et al., 2011). Canditatus Magnetobacterium casensis shares similar morphology and $98.2 \%$ of the sequence identity of the 16S rRNA gene with $C a$. Magnetobacterium bavaricum. Analysis of the draft genome sequence of $C a$. Magnetobacterium casensis reveals an autotrophic lifestyle. Pathways involved in denitrification, sulfur oxidation and sulfate reduction have also been predicted, which indicates its considerable capacity to adapt to variable environments (Lin et al., 2014).

The third identified Nitrospirae MTB was a small rod-shaped bacterium collected from sediment of Waller See, in Germany and named MHB-1 (Flies et al., 2005). This organism contains a single bundle of multiple chains of magnetosomes with bullet-shaped magnetite crystals. The fourth reported Nitrospirae MTB is a moderately thermophilic magnetotactic bacterium, HSMV-1, which remained viable and motile in samples kept between 25 and $62^{\circ} \mathrm{C}$ for several months (Lefèvre et al., 2010). HSMV-1 cells are small vibrios and biomineralize a single chain of bullet-shaped magnetosomes. Two Nitrospirae MTB phylotypes named LO-1 and MWB-1 were identified from southern Nevada, USA and Beijing, China, respectively (Lefevre et al., 2011; Lin et al., 2012a). The two species have 6\% phylogenetic divergence and possess a similar relatively big $(\sim 3 \times 2.5 \mu \mathrm{m})$ spherical morphology with bullet-shaped magnetosomes and 4-7 bundles of magnetosome chains parallel to the long axis of cells. Interestingly, the six described Nitrospirae MTB phylotypes have distinct cell 
appearance and different magnetosome arrangement including single chain, multiple chains with single bundle or multiple bundles. The obvious common feature is their bullet-shape magnetite magnetosomes. Another common property of the reported Nitrospirae MTB is that they dwell in low salinity habitats ( $<3$ ppt) (Spring et al., 1993; Flies et al., 2005; Lefevre et al., 2011; Lin et al., 2012a; Lin et al., 2012b). Therefore, it has been proposed that salinity might influence the distribution of MTB affiliated with Nitrospirae phylum (Lin et al., 2012b). Here, we identified two novel Nitrospirae MTBs species and expand the distribution of Nitrospirae MTB to marine ecosystems.

\section{Results and discussion}

\section{Morphology and swimming behaviour of black-brown cocci and ivory-white cocci}

Differential interference contrast (DIC) microscopic examination showed the morphology of black-brown giant cocci (BGC) (Fig. 1 A, black arrows), ivory-white giant cocci (WGC) (Fig. 1 A, white arrows) and small cocci of MTB collected from the sediments in Six-Fours les Plages. BGC have a rough surface appearance with an average length of $5.39 \pm 0.72 \mu \mathrm{m}$ and an average width of $4.04 \pm 0.33 \mu \mathrm{m}(\mathrm{n}=62)$. WGC have a smoother cell surface and a similar cell size with an average length of $5.21 \pm 0.66 \mu \mathrm{m}$ and an average width of $3.99 \pm 0.30$ $\mu \mathrm{m}(\mathrm{n}=62)$ (B in Figure 1). During sample preparation for DNA amplification and sequencing, we observed that BGC cells began to disintegrate 5 min following their transfer from seawater to PBS buffer, and small granules were released from the cells (C1 in Figure 1). In contrast, WGC cells shrunk and became smaller in PBS buffer (C2 in Figure 1). Further analysis showed that approximately $50 \%$ of the BGC and WGC cells remained motile and 
accumulated at the north edge of the cell suspension droplets where the salinity was decreased from 35 to $16 \mathrm{ppt}$ by dilution with deionized water. The number of cells at the edge of the droplets decreased as the dilution increased, and the BGC and WGC cells almost disappeared completely from the droplets at $13 \mathrm{ppt}$ salinity. This observation suggests a sensitivity of the marine cells to the freshwater conditions. BGC and WGC also exhibited different fluorescent properties. When excited with green light $(510-550 \mathrm{~nm}), \mathrm{BGC}$ emitted red fluorescence while no auto-fluorescence signals were observed in WGC under these conditions (D2 in Figure1). BGC emitted stronger yellow auto-fluorescence than WGC under blue light $(450-480 \mathrm{~nm})$ excitation (D3 in Figure1). Similar intensity of green light was emitted by both BGC and WGC when excited by violet light (400-410 nm) (D4 in Figure 1). WGC emitted stronger blue fluorescence under ultraviolet light (330-385 nm) compared to BGC (D5 in Figure 1). Illumination at these wavelengths had no impact on the motility of these MTB cells. Lack of culture samples prevents us from performing detailed spectrophotometry analysis to characterize the nature of the fluorescent molecules.

Most of the BGC and WGC exhibited north-seeking magnetotaxis behavior, i.e. swimming along the applied magnetic field lines, whereas $10 \%$ of cells were found to be south-seeking. Cells demonstrated a dynamic motility in the hanging drops for approximately 30 min then began to slow down gradually and stopped swimming at the end of one hour. The average swim velocities of BGC and WGC cells were $126.3 \pm 45.0 \mu \mathrm{m} \cdot \mathrm{s}^{-1}(\mathrm{n}=52)$ and $148.9 \pm$ $28.0 \mu \mathrm{m} \cdot \mathrm{s}^{-1}(\mathrm{n}=54)$, respectively ( $\mathrm{E}$ in Figure 1$)$. The corresponding maximal swim speeds of BGC $\left(225.4 \mu \mathrm{m} \cdot \mathrm{s}^{-1}\right)$ and WGC $\left(188.6 \mu \mathrm{m} \cdot \mathrm{s}^{-1}\right)$ were slower than the closest freshwater relative MTB MWB-1 $\left(260 \mu \mathrm{m} \cdot \mathrm{s}^{-1}\right)$. 
In the environmental samples analyzed, BGC cells were the dominant MTB while WGC were approximately 4-fold less abundant as estimated by direct counting of the two morphotypes in droplets (Experimental Procedures and Supporting Information Figure S1). We collected 8 WGC cells using the magnetic micro-sorting approach, and performed Whole Genome Amplification to get sufficient template DNA for further 16S rRNA gene amplification and cloning. We obtained 24 sequences of $16 \mathrm{~S}$ rRNA genes that shared $>99.3 \%$ 
Lin et al. suggested that the magnetosome biomineralization and magnetotaxis was present before the separation of the Nitrospirae and Proteobacteria phyla, which was between $\sim 3.38$ and 3.21 Ga (Lin et al., 2017b). However, it remains unclear whether the acquisition of magnetosome island (MAI), that encodes essential proteins for magnetosome biosynthesis in the genome, occurred before or after the divergence between freshwater and marine Nitrospirae MTB. It is equally possible that the spherical cells of Nitrospirae phylum got MAI by horizontal gene transfer (HGT) then adapted into two different habitats or that they diverged into different habitats then acquired MAI separately. Future genomic comparison studies should provide answers to elucidate the evolutionary mechanism.

\section{Corroboration of authenticity of giant cocci by fluorescence in situ hybridization}

The BGC authenticity of OTU-2 was inferred from the fact that BGC is the most abundant morphotype in the analyzed samples. To corroborate this inference we designed a specific probe based on the conserved OTU-2 sequence and performed fluorescence in situ hybridization (FISH) analysis. As shown in Figure 3 A2, the universal bacterial probe hybridized with all the MTB, while the specific probe hybridized only with black-brown giant cocci. The WGC cells only hybridized with the bacteria universal probe and were used as a negative control. Therefore, the OTU-2 really corresponds to the abundant BGC morphotype.

We also confirmed the authenticity of the OTU-1 to the scarce WGC. A probe specific to the conserved sequences of OTU-1 was designed. We found that the specific probe hybridized only with the WGC morphotype while the universal probe hybridized with all bacterial cells including BGC that served as the negative control (Fig. 3, B1). After FISH identification, the 
same cover-slips were observed under scanning electron microscopy (SEM). As shown in B3 of Fig. 3, more than 8 bundles of multiple magnetosome chains aligned parallel to the long axis and close to the outer surface in the WGC cell. Correlative FISH-SEM analysis not only allowed identification of the OTU but also revealed characteristics of the magnetosome chains.

\section{Magnetosome and element composition analyses}

After corroborating their authenticities, we analyzed the magnetosome chains and element composition in BGC and WGC. Under DIC microscopy BGC appeared to contain brown granules inside the cells while WGC had a smooth appearance (Fig. 4A). Observations using High-angle annular dark-field scanning transmission electron microscopy (HAADF-STEM) revealed that the BGC cells were filled with electron dense granules rich in sulfur (Fig. 4B, top cell with white inclusions, and 4C in red color) in addition to the magnetosomes chains (Fig. 4C green color). Further energy-dispersive X-ray spectroscopy (XEDS) analysis confirmed that the granules were rich in sulfur, the most abundant element in BGC cells but barely detectable in WGC (Fig. 4, D2 versus D1). The magnetosomes in WGC were arranged in bundles of multiple chains as revealed by FISH-SEM analysis. We attempted to identify the magnetosome crystals inside of BGC and WGC cells by electron diffraction or high-resolution electron microscopy without success due to the thickness of the cells. Although we identified isolated bullet-shaped crystals as magnetites on grids, but it is uncertain if they were from BGC or WGC because environmental samples containing other MTBs were used. We performed STEM-XEDS elemental map (Fig. 4C) and XEDS spectrum 
analyses (Fig. 4B, E1, E2, F1 and F2), and unambiguously showed that the crystals in BGC and WGC cells are iron-oxide minerals, which is consistent with the finding of only magnetite minerals in Nitrospirae MTB magnetosomes in the literature.

One essential difference between BGC and WGC is that BGC contain numerous sulfur-rich inclusions. Intracellular sulfur globules were also detected in Nitrospirae magnetotactic bacteria $C a$. Magnetobacterium bavaricum, LO-1, MWB-1 and MYR-1 (Jogler et al., 2010; Li et al., 2010; Lefevre et al., 2011; Lin et al., 2012a; Li et al., 2016), and those in the $C a$. Magnetobacterium bavarium are present in the form of cyclo-octa sulfur (S8) (Eder et al., 2014). Intracellular deposition of sulfur may serve as an electron donor or an electron acceptor to provide energy for bacterial growth (Cox et al., 2002). We observed that BGC became the dominant population during storage under laboratory conditions and were able to survive for more than ten months while maintaining vivacious magnetotactic motility. Therefore, it is plausible that the metabolism of intracellular sulfur-rich inclusions might contribute to the long-term survival of BGC under laboratory conditions.

In conclusion, we reported the discovery of two novel marine species of Nitrospirae MTB and their morphology and structural differences. Future genomic analysis would be helpful to understand the evolution and adaptation mechanisms of Nitrospirae magnetotactic bacteria to freshwater and marine environments.

\section{Experimental Procedures}

\section{Sediment collection and MTB enrichment}


Mediterranean Sea, Southern France. The site was characterized by a salinity of $\sim 35 \mathrm{ppt}, \mathrm{pH}$ of 7.5 and a temperature of $19^{\circ} \mathrm{C}$ at the time of sampling. Collected sediments were quickly transferred to $1000 \mathrm{ml}$ plastic bottles, covered with approximately $600 \mathrm{ml}$ of in situ seawater, transported to the laboratory, and incubated at room temperature without disturbance.

Black-brown giant cocci cells (BGC) in the sediment were magnetically enriched using the race-track method (Wolfe et al., 1987; Zhu et al., 2010). The 16S rRNA genes were directly amplified from the collections. White giant cocci cells (WGC) were further collected by magnetic micro-sorting using a Transfer Man ONM-2D micromanipulator and an IM-9B CellTram Oil manual hydraulic pressure-control system installed on an IX51 microscope equipped with a $40 \times$ LD objective (Zhang et al., 2014). WGC cells were directly transferred to $3 \mu 1$ of PBS of the REPLI-g Single Cell Kit (QIAGEN). The PBS-suspended sample was frozen and thawed repeatedly, and the whole genome amplification (WGA) reaction was carried out using the REPLI-g Single Cell Kit (Chen et al., 2015). The 16S rRNA gene sequences were obtained as previously described by Chen et al. (Chen et al., 2015).

\section{Phylogenetic analysis}

The 16S rDNA sequences were aligned using CLUSTAL W multiple alignment software. Sequence identities were calculated with the BIOEDIT software. The phylogenetic tree was constructed via the neighbor-joining method using MEGA VERSION (Kumar et al., 2016), and boot-strap values were calculated from a 1000 replicates.

\section{Optical microscopy}


The morphologies and motilities of the enriched MTB cells were examined under DIC microscopy (Olympus BX51 equipped with a DP71 camera; Olympus) using the hanging-drop method in an applied magnetic field. The ratio between BGC and WGC was estimated by counting the two morphotypes of cells at the edge of the droplets as shown in Supporting Information Figure S1. To analyze the sensitivity to the osmolality the collected cell suspensions were diluted with deionized water before observing their magnetotactic behavior in the droplets. The trajectories of the cells were obtained using the dark-field mode with an increased exposure time. To assess auto-fluorescence, fluorescence mirror units were used to expose the cells to green light $(510-550 \mathrm{~nm})$, blue light $(450-480 \mathrm{~nm})$, violet light (400-410 nm) and UV light (330-385 nm) with the corresponding filter sets of Olympus.

FISH analyses

BGC-specific probe (5'-ATACTTAATCTTCCGCAGACC-3') and WGC-specific probes (5'-GGTTCCACACCGAAGCATT-3') were designed according to the conserved 16S rRNA gene sequences of OTU-2 (GenBank number MK073023) and OTU-1 (GenBank number MK203828), respectively and evaluated for their specificity by using the online probe

evaluation

tools

Ribosomal

Database

Project

(RDP)

251 https://rdp.cme.msu.edu/probematch/search.jsp (Cole et al., 2009; Cole et al., 2014). The

BGC- and WGC-specific probes were synthesized and fluorescently labeled with the hydrophilic sulfoindocyanine dye Cy3 at the 5' end. The universal bacterial probe EUB338 (5'-GCTGCCTCCCGTAGGAGT-3') was fluorescently labeled with fluorescein phosphoramidite 200 FAM at the 5' end and used as a positive control for FISH (Amann et al., 
1990).

\section{Correlative FISH-SEM analyses}

The FISH and correlative FISH-SEM analyses were carried out as described by Li et al.

(Li et al., 2017). The same FISH coverslips of WGC were treated for SEM observations using

a Zeiss Ultra-55 field-emission gun SEM (Carl Zeiss) operating at $5 \mathrm{kV}$ (Li et al., 2017).

\section{HAADF-STEM elemental composition analysis}

Magnetosome biomineralization was investigated using a JEM-2100F microscope (JEOLLtd) operating at $200 \mathrm{kV}$ equipped with a field emission gun, an ultra-high resolution (UHR) pole piece and a Gatan energy filter (GIF2001) system (Gatan, Inc.). HAADF-STEM was used for Z-contrast imaging. Chemical compositional analysis was performed by EDXS elemental mapping in the HAADF-STEM mode. By using this method, nanometer-scale spatial distribution of most elements can be determined, and the relative abundance of each element at a certain location can be semi-quantitatively calculated (Li et al., 2017).

\section{Acknowledgments}

We are grateful to H. Zhang and F.X. Wang for their assistance in the coordinated FISH-SEM experiments. X.X. Qian is grateful for her fellowship provided by the Chinese

272 Scholarship Council. This work was supported by grants 41776131 and U1706208 from NSFC, grants 2016YFC0302502_02 and 2018YFC0309904 from National Key R\&D

274 Program of China, a grant XDB06010203 from the Strategic Priority Research Program, by a 
grant from the Excellence Initiative of Aix-Marseille University - A*Midex, a French "Investissements d'Avenir" programme, and grant from CNRS for LIA-MagMC. The FISH-SEM experiments were performed at the IGGCAS, Beijing, China and were supported by a NSFC grant 41522402 .

\section{Conflict of interest}

The authors declare that they have no conflict of interest

\section{References}

Amann, R.I., Krumholz, L., and Stahl, D.A. (1990) Fluorescent-oligonucleotide probing of whole cells for determinative, phylogenetic, and environmental studies in microbiology. $J$ bacteriol 172: 762-770.

Bazylinski, D.A., and Frankel, R.B. (2004) Magnetosome formation in prokaryotes. Nat Rev Microbiol 2: 217.

Blakemore, R. (1975) Magnetotactic bacteria. Science 190: 377.

Chen, Y.R., Zhang, R., Du, H.J., Pan, H.M., Zhang, W.Y., Zhou, K. et al. (2015) A novel species of ellipsoidal multicellular magnetotactic prokaryotes from Lake Yuehu in China. Environ Microbiol 17: 637-647.

Cole, J.R., Wang, Q., Fish, J.A., Chai, B., McGarrell, D.M., Sun, Y. et al. (2014) Ribosomal Database Project: data and tools for high throughput rRNA analysis. Nucleic Acids Res 42: D633-D642.

Cole, J.R., Wang, Q., Cardenas, E., Fish, J., Chai, B., Farris, R.J. et al. (2009) The Ribosomal 
Database Project: improved alignments and new tools for rRNA analysis. Nucleic Acids Res

37: D141-D145.

Cox, B.L., Popa, R., Bazylinski, D.A., Lanoil, B., Douglas, S., Belz, A. et al. (2002)

Organization and Elemental Analysis of P-, S-, and Fe-rich Inclusions in a Population of

Freshwater Magnetococci. Geomicrobiol J 19: 387-406.

Eder, S.H.K., Gigler, A.M., Hanzlik, M., and Winklhofer, M. (2014) Sub-micrometer-scale mapping of magnetite crystals and sulfur globules in magnetotactic bacteria using confocal Raman micro-spectrometry. PloS one 9: e107356-e107356.

Flies, C.B., Peplies, J., and Schüler, D. (2005) Combined Approach for Characterization of

Uncultivated Magnetotactic Bacteria from Various Aquatic Environments. Appl Environ Microbiol 71: 2723-2731.

Jogler, C., Wanner, G., Kolinko, S., Niebler, M., Amann, R., Petersen, N. et al. (2011) Conservation of proteobacterial magnetosome genes and structures in an uncultivated member of the deep-branching Nitrospira phylum. Proc Natl Acad Sci U S A 108: 1134-1139.

Jogler, C., Niebler, M., Lin, W., Kube, M., Wanner, G., Kolinko, S. et al. (2010) Cultivation-independent characterization of 'Candidatus Magnetobacterium bavaricum' via ultrastructural, geochemical, ecological and metagenomic methods. Environ Microbiol 12: 2466-2478.

Kumar, S., Stecher, G., and Tamura, K. (2016) MEGA7: Molecular Evolutionary Genetics Analysis Version 7.0 for Bigger Datasets. Mol Biol Evol 33: 1870-1874.

Lefèvre, C.T., Abreu, F., Schmidt, M.L., Lins, U., Frankel, R.B., Hedlund, B.P., and Bazylinski, D.A. (2010) Moderately Thermophilic Magnetotactic Bacteria from Hot Springs 
in Nevada. Appl Environ Microbiol 76: 3740-3743.

Lefevre, C.T., and Bazylinski, D.A. (2013) Ecology, diversity, and evolution of magnetotactic bacteria. Microbiol Mol Biol Rev 77: 497-526.

Lefevre, C.T., Frankel, R.B., Abreu, F., Lins, U., and Bazylinski, D.A. (2011) Culture-independent characterization of a novel, uncultivated magnetotactic member of the Nitrospirae phylum. Environ Microbiol 13: 538-549.

Li, J., Menguy, N., Gatel, C., Boureau, V., Snoeck, E., Leroy, E., and Patriarche, G. (2016) Crystal growth of bullet-shaped magnetite in magnetotactic bacteria of the Nitrospirae phylum. J Royal Soc Interface 12: 103.

Li, J., Zhang, H., Menguy, N., Benzerara, K., Wang, F., Lin, X. et al. (2017) Single-Cell Resolution of Uncultured Magnetotactic Bacteria via Fluorescence-Coupled Electron Microscopy. Appl Environ Microbiol 83: e00409-00417.

Li, J., Pan, Y., Liu, Q., Yu-Zhang, K., Menguy, N., Che, R. et al. (2010) Biomineralization, crystallography and magnetic properties of bullet-shaped magnetite magnetosomes in giant rod magnetotactic bacteria. Earth Planet Sci Lett 293: 368-376.

Lin, W., Li, J., and Pan, Y. (2012a) Newly isolated but uncultivated magnetotactic bacterium of the phylum Nitrospirae from Beijing, China. Appl Environ Microbiol 78: 668-675.

Lin, W., Pan, Y., and Bazylinski, D.A. (2017a) Diversity and ecology of and biomineralization by magnetotactic bacteria. Environ Microbiol Rep 9: 345-356.

Lin, W., Wang, Y., Li, B., and Pan, Y. (2012b) A biogeographic distribution of magnetotactic bacteria influenced by salinity. ISME J 6: 475-479.

Lin, W., Zhang, W., Zhao, X., Roberts, A.P., Paterson, G.A., Bazylinski, D.A., and Pan, Y. 
(2018) Genomic expansion of magnetotactic bacteria reveals an early common origin of magnetotaxis with lineage-specific evolution. ISME J 12: 1508-1519.

Lin, W., Deng, A., Wang, Z., Li, Y., Wen, T., Wu, L.-F. et al. (2014) Genomic insights into the uncultured genus 'Candidatus Magnetobacterium' in the phylum Nitrospirae. ISME J 8: 2463. Lin, W., Paterson, G.A., Zhu, Q., Wang, Y., Kopylova, E., Li, Y. et al. (2017b) Origin of microbial biomineralization and magnetotaxis during the Archean. Proc Natl Acad Sci 114: 2171-2176.

Spring, S., Amann, R.I., Ludwig, W., Schleifer, K., van Gemerden, H., and Petersen, N. (1993) Dominating Role of an Unusual Magnetotactic Bacterium in the Microaerobic Zone of a Freshwater Sediment. Appl Environ Microbiol 59: 2397-2403.

Uebe, R., and Schüler, D. (2016) Magnetosome biogenesis in magnetotactic bacteria. Nat Rev Microbiol 14: 621.

Wolfe, R.S., Thauer, R.K., and Pfennig, N. (1987) A ‘capillary racetrack’ method for isolation of magnetotactic bacteria. FEMS Microbiol Lett 45: 31-35.

Zhang, R., Chen, Y.R., Du, H.J., Zhang, W.Y., Pan, H.M., Xiao, T., and Wu, L.F. (2014) Characterization and phylogenetic identification of a species of spherical multicellular magnetotactic prokaryotes that produces both magnetite and greigite crystals. Res Microbiol 165: 481-489.

Zhu, K., Pan, H., Li, J., Yu-Zhang, K., Zhang, S.-D., Zhang, W. et al. (2010) Isolation and characterization of a marine magnetotactic spirillum axenic culture QH-2 from an intertidal zone of the China Sea. Res Microbiol 161: 276-283. 

giant cocci (WGC).

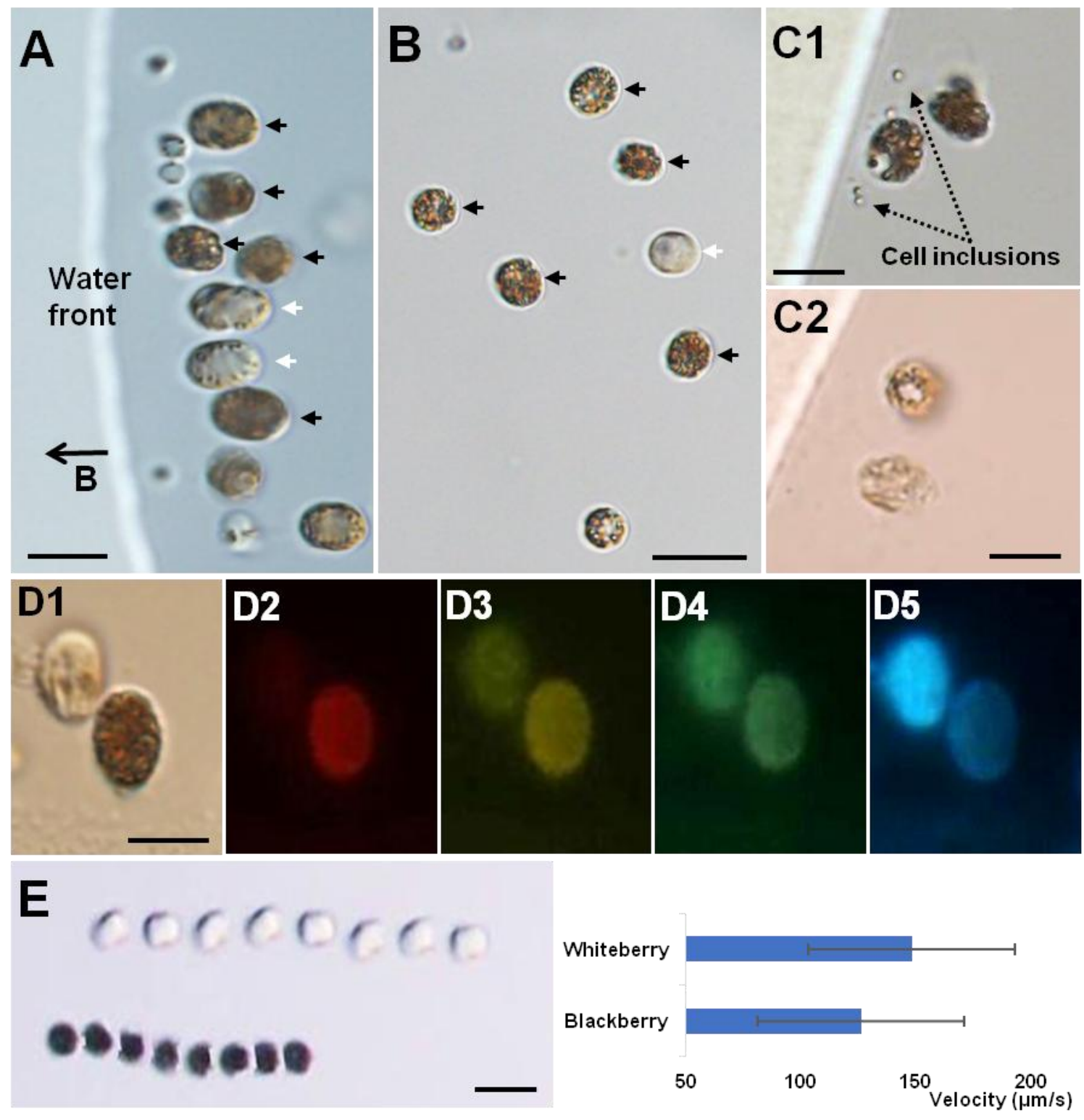

(A) Differential interference contrast (DIC) micrograph shows magnetotaxis and accumulation

of BGC (indicated by black arrows) and WGC (white arrows) at the north edge in a droplet. The

big black arrow B indicates the direction of the magnetic field. (B) DIC micrograph shows the rough surface of BGC and smooth surface of WGC. (C) DIC micrograph shows both BGC 

shows auto-fluorescence of BGC and WGC excited by light at different wavelengths. (D1)

Figure 2. Phylogenetic tree of 16S rRNA gene sequences from Nitrospirae MTB.

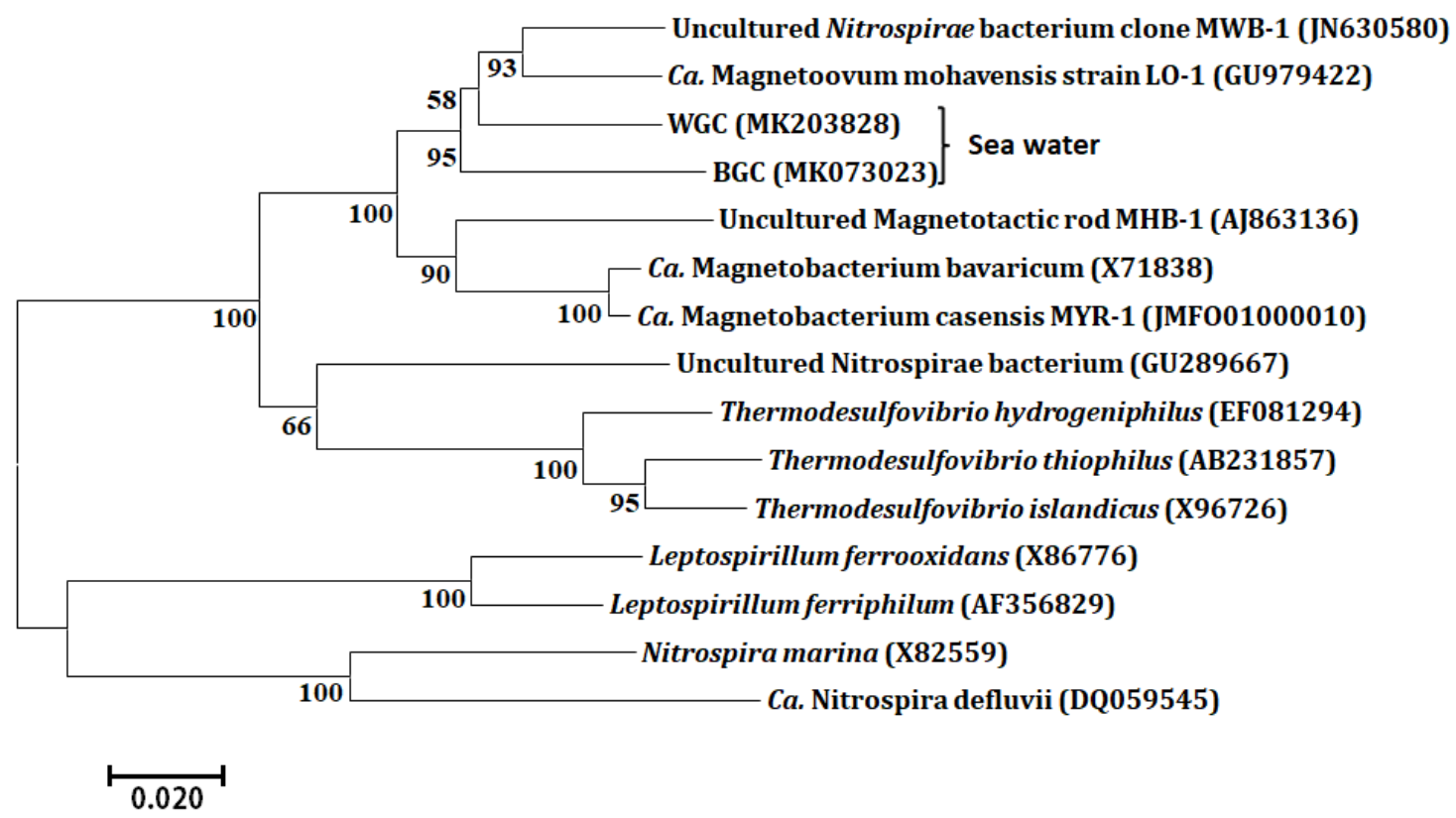

All currently characterized Nitrospirae MTB and some typical Nitrospirae non-MTBs were 

given in parentheses. Scale bar represents $2 \%$ sequence divergence.

\section{Figure 3. FISH identification of magnetotactic giant cocci.}
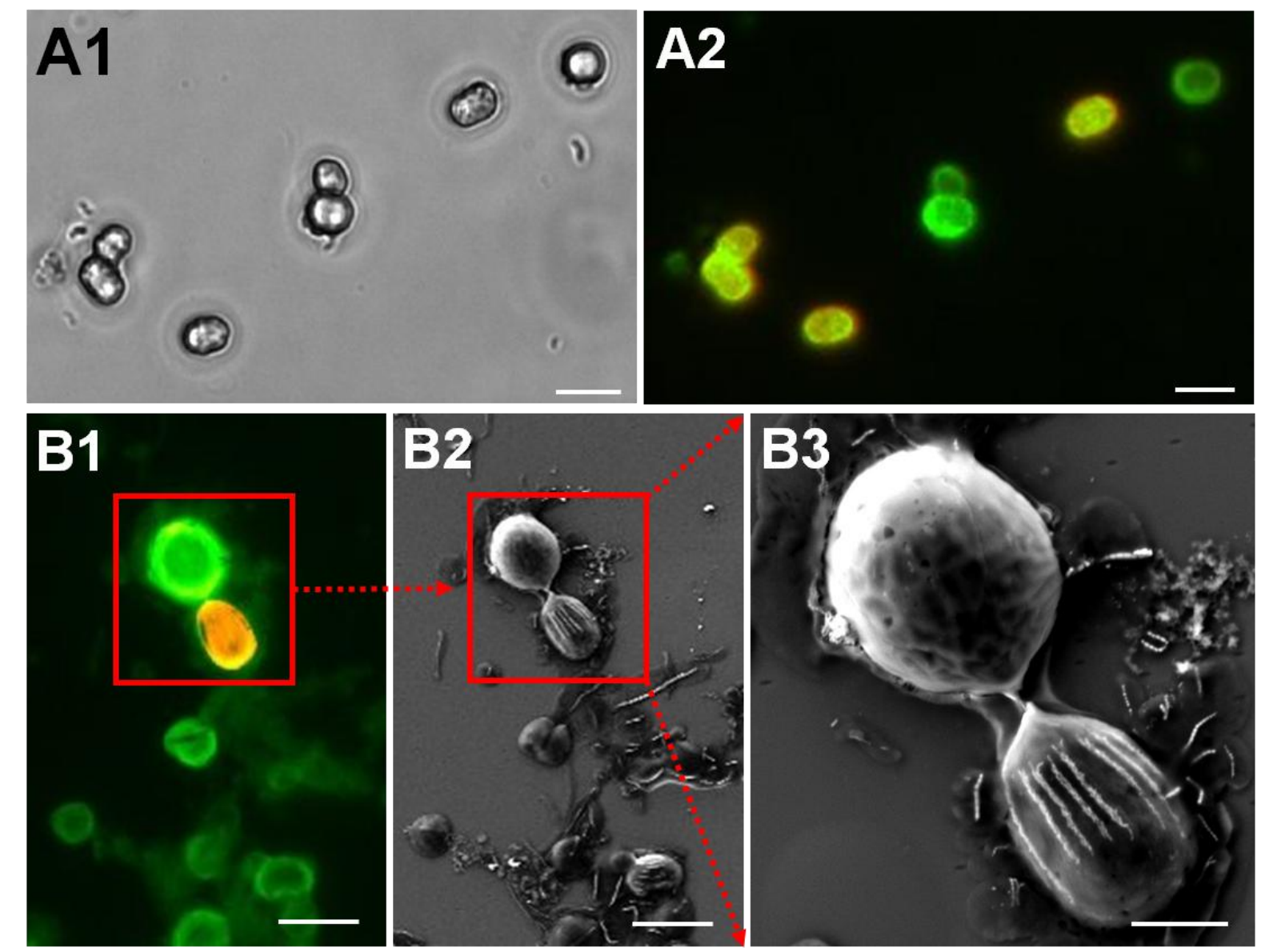

Optical micrograph (A1) and fluorescence micrograph (A2) show BGC cells that were in situ hybridized (FISH) with the 5'-FAM-labeled universal bacterial probe EUB338 and the universal bacterial probe and yellow color shows the cell hybridized with both the universal bacterial probe and the BGC-specific probe. (B) Correlative FISH - SEM microscopic analysis of the WGC. (B1) In fluorescence micrograph, green color indicates bacteria hybridized only with universal bacterial probe EUB338 and yellow color shows the cell hybridized with both the universal bacterial probe and the 5'-Cy3-labeled WGC-specific probe. (B2) Correlated 
SEM image of image B1. (B3) Correlated SEM image of the same red frame field in image B2 WGS. The scale bars: $5 \mu \mathrm{m}$ in A1, A2, B1, B2. $2 \mu \mathrm{m}$ in B3. 

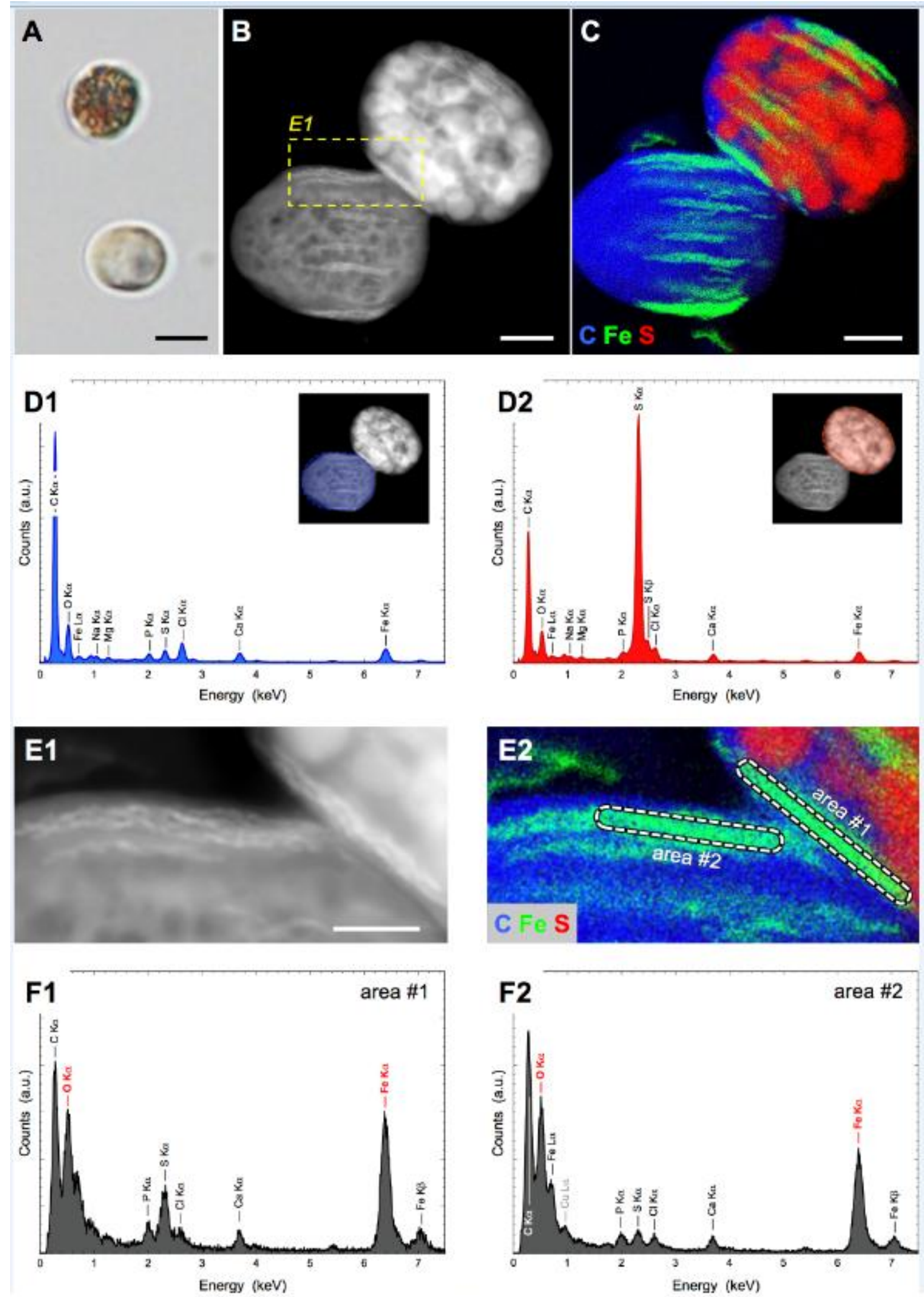

A) DIC image showed overall morphology difference between BGC (top cell) and WGC (bottom cell). (B) STEM-HAADF image of BGC and WGC revealed multiple bundles of 

scale bars: $5 \mu \mathrm{m}$ in $\mathrm{A}, 1 \mu \mathrm{m}$ in $\mathrm{B}$ and $\mathrm{C}, 0.5 \mu \mathrm{m}$ in $\mathrm{E} 1$.

magnetosome chains in both and granules filled in BGC, dashed rectangle is related to the area shown in E1. (C) STEM-XEDS elemental map of BGC and WBC revealing the presence of sulfur (red) and iron (green) overlaid with granules in BGC and iron-oxide crystals of magnetosomes in both BGC and WGC. (D1) XEDS spectrum corresponding to WGC showing that carbon is the most abundant element. (D2) XEDS spectrum corresponding to BGC showing that sulfur is the most abundant element. (E1) STEM-HAADF image related to the selected area in (A) and corresponding elemental map (E2). (F1) and (F2) represent XEDS spectra of the magnetosome chains in the selected areas \#1 and \#2 (in E2) in WBC and BGC cells, respectively. These spectra indicate the iron-oxide nature of magnetosome crystals. The 